\title{
FIRST PASSAGE PROBLEMS FOR ASYMMETRIC WIENER PROCESSES
}

\author{
MARIO LEFEBVRE, ${ }^{*}$ École Polytechnique de Montréal
}

\begin{abstract}
The problem of computing the moment generating function of the first passage time $T$ to $a>0$ or $-b<0$ for a one-dimensional Wiener process $\{X(t), t \geq 0\}$ is generalized by assuming that the infinitesimal parameters of the process may depend on the sign of $X(t)$. The probability that the process is absorbed at $a$ is also computed explicitly, as is the expected value of $T$.
\end{abstract}

Keywords: Hitting time; Brownian motion; Kolmogorov equation

2000 Mathematics Subject Classification: Primary 60J70

Secondary $60 \mathrm{~J} 65$

\section{Introduction}

In a relatively recent paper, Chong et al. (2000) obtained results about cover times for asymmetric random walks. The cover time is the number of steps taken by the random walk until its range is of a given magnitude. They also studied this problem for Brownian motions.

Carlsund (2003) considered the cover time problem for sign-dependent random walks (that is, simple random walks for which the one-step transition probabilities may be different on the positive and negative half-lines) with exponential holding times. In addition, she computed the moment generating function of the time of first passage to $a>0$ or $-b<0$, assuming that the process starts at the origin. By using an appropriate scaling, she obtained the moment generating function of the same first passage time for an asymmetric Brownian motion (also starting at the origin).

Here we generalize and extend Carlsund's results for asymmetric Brownian motions $\{X(t), t \geq 0\}$. In Section 2, the moment generating function of the first passage time $T(x)$ to $a>0$ or $-b<0$ will be computed explicitly for any starting value $X(0)=x$ of the process in the interval $(-b, a)$. We will also calculate the probability that $X(T(x))=a$ and the expected value of $T(x)$. A few remarks conclude the paper, in Section 3.

\section{Explicit results}

Let $\{W(t), t \geq 0\}$ be a Wiener process with infinitesimal parameters $\mu$ and $\sigma^{2}$, and define

$$
\tau(w)=\inf \{t>0: W(t)=a \text { or } W(t)=-b \mid W(0)=w\},
$$

where $w \in(-b, a)$. The distribution of the random variable $\tau(w)$ is well known, as is the probability $\mathrm{P}[W(\tau(w))=a]$ (see, for example, Cox and Miller (1965, p. 222, p. 233)).

In this note, $\{X(t), t \geq 0\}$ is a Wiener process with infinitesimal parameters $\mu_{+}$and $\sigma_{+}^{2}$ when $X(t)>0$, and $\mu_{-}$and $\sigma_{-}^{2}$ when $X(t)<0$. Moreover, when the process hits the origin, it

Received 30 March 2005; revision received 1 September 2005.

* Postal address: Département de Mathématiques et de Génie Industriel, École Polytechnique, C.P. 6079, Succursale Centre-ville, Montréal, Québec, Canada H3C 3A7. Email address: mario.lefebvre@ polymtl.ca 
goes right with probability $p$ and left with probability $q:=1-p$. Thus, $\{X(t), t \geq 0\}$ could be called a sign-dependent Wiener process. We prefer the term asymmetric Wiener process, because we could actually assume that its infinitesimal parameters change when it crosses any given barrier, not necessarily the origin.

One way to obtain the process described above is to start with a sign-dependent random walk such that, for every time unit $\delta_{+} t$, from any state $k \delta_{+} x$ (with $k>0$ ) it moves $\delta_{+} x$ units to the right with probability $p_{+}$and it moves $\delta_{+} x$ units to the left with probability $q_{+}$. When $k<0$, $\delta_{+}, p_{+}$, and $q_{+}$are replaced by $\delta_{-}, p_{-}$, and $q_{-}$, respectively. Moreover, when the random walk hits the origin, it goes right $\delta_{+} x$ units with probability $p$ and it goes left $\delta_{-} x$ units with probability $q$. Then, taking the limit as $\delta_{ \pm} t$ and $\delta_{ \pm} x$ decrease to zero, with $\left(\delta_{ \pm} x\right)^{2} / \delta_{ \pm} t=\sigma_{ \pm}^{2}$, we get the asymmetric Wiener process, assuming that the difference $p_{ \pm}-q_{ \pm}$is equal to $\left(\mu_{ \pm} / \sigma_{ \pm}^{2}\right) \delta_{ \pm} x$.

The asymmetric Wiener process is related to the so-called skew Brownian motion introduced by Itô and McKean (1974, p. 115); see also Harrison and Shepp (1981). In the case of a skew Brownian motion, the process behaves like a standard Brownian motion with a reflecting barrier at the origin, except that when it hits the origin it is reflected to the positive part of the real axis with probability $p$ and it is reflected to the negative part of the real axis with probability $1-p$, where $0<p<1$.

We now present an application of the asymmetric Wiener process. Suppose that $X(t)$ is the price of a stock or a commodity, such as gold or oil, at time $t$. In practice, when this price reaches a certain threshold, it often becomes much more volatile. For example, if we accept that a Wiener process could serve as a model for the price of gold above $\$ 400$, or the price of a barrel of oil above $\$ 50$, it is reasonable to assume that the variance parameter, in particular, is larger when the price of the commodity is above the mentioned threshold.

Next, let

$$
T(x)=\inf \{t>0: X(t)=a \text { or } X(t)=-b \mid X(0)=x\},
$$

where $a>0, b>0$, and $x \in(-b, a)$, and let

$$
M(x)=\mathrm{E}\left[\mathrm{e}^{-\alpha T(x)}\right]
$$

where $\alpha$ is a positive constant. The moment generating function $M(x)$ satisfies the Kolmogorov backward equation

$$
\frac{1}{2} \sigma_{ \pm}^{2} M^{\prime \prime}(x)+\mu_{ \pm} M^{\prime}(x)=\alpha M(x),
$$

where the ' + ' sign is chosen when $x>0$, the ' - ' $\operatorname{sign}$ is chosen when $x<0$, and a prime denotes differentiation with respect to $x$.

Ovaskainen and Cornell (2003) considered Kolmogorov equations of the type shown in (2), and gave the appropriate boundary conditions at the origin when the corresponding diffusion process is obtained as the limiting process of a particular continuous-time, discrete-state stochastic process. They also gave general results about hitting probabilities and occupancy times for multidimensional diffusion processes with possible discontinuities across interior boundaries. These processes have applications in biology.

Now, to compute the quantities of interest for the asymmetric Wiener process, we will modify it as follows. We assume that when the process hits the origin, it jumps to $\delta>0$ with probability $p$ and to $-\delta$ with probability $q$, where $\delta$ is smaller than $a$ and $-b$. Let $T_{\delta}(x)$ be the random variable corresponding to $T(x)$ in this case and let $M_{\delta}(x)$ be its moment generating function. We can show, in particular, that

$$
M(x)=\lim _{\delta \downarrow 0} M_{\delta}(x)
$$


We define

$F$ : the event that the process $X(t)$ hits $a$ before 0 from $x \in(0, a)$,

$G$ : the event that the process $X(t)$ hits $-b$ before 0 from $x \in(-b, 0)$,

and

$T_{x, a}^{+}$: the time taken by $X(t)$ to go from $x \in(0, a)$ to $a$ without hitting 0 ,

$T_{x, 0}^{+}$: the time taken to go from $x \in(0, a)$ to 0 without hitting $a$,

$T_{x,-b}^{-}$: the time taken to go from $x \in(-b, 0)$ to $-b$ without hitting 0 ,

$T_{x, 0}^{-}$: the time taken to go from $x \in(-b, 0)$ to 0 without hitting $-b$.

\subsection{Moment generating function of $T(x)$}

We may write, for $0<x<a$,

$$
\begin{aligned}
M_{\delta}(x) & :=\mathrm{E}\left[\mathrm{e}^{-\alpha T_{\delta}(x)}\right] \\
& =\mathrm{E}\left[\mathrm{e}^{-\alpha T_{\delta}(x)} \mid F\right] \mathrm{P}[F]+\mathrm{E}\left[\mathrm{e}^{-\alpha T_{\delta}(x)} \mid F^{\mathrm{c}}\right] \mathrm{P}\left[F^{\mathrm{c}}\right] \\
& =\mathrm{E}\left[\mathrm{e}^{\left.-\alpha T_{x, a}^{+}\right]} \mathrm{P}[F]+\mathrm{E}\left[\mathrm{e}^{-\alpha\left(T_{x, 0}^{+}+T_{\delta}(0)\right)}\right] \mathrm{P}\left[F^{\mathrm{c}}\right]\right. \\
& =M_{x, a}+M_{x, 0} M_{\delta}(0) \quad \text { (by independence), }
\end{aligned}
$$

where

$$
M_{x, a}:=\mathrm{E}\left[\mathrm{e}^{-\alpha T_{x, a}^{+}}\right] \mathrm{P}[F] \quad \text { and } \quad M_{x, 0}:=\mathrm{E}\left[\mathrm{e}^{-\alpha T_{x, 0}^{+}}\right] \mathrm{P}\left[F^{\mathrm{c}}\right] .
$$

Similarly, we define, for $-b<x<0$,

$$
N_{x,-b}=\mathrm{E}\left[\mathrm{e}^{-\alpha T_{x,-b}^{-}}\right] \mathrm{P}[G] \quad \text { and } \quad N_{x, 0}=\mathrm{E}\left[\mathrm{e}^{-\alpha T_{x, 0}^{-}}\right] \mathrm{P}\left[G^{\mathrm{c}}\right] .
$$

Then, we have

$$
M_{\delta}(0)=p M_{\delta}(\delta)+q M_{\delta}(-\delta),
$$

with

$$
\begin{aligned}
M_{\delta}(\delta) & =M_{\delta, a}+M_{\delta, 0} M_{\delta}(0), \\
M_{\delta}(-\delta) & =N_{-\delta,-b}+N_{-\delta, 0} M_{\delta}(0) .
\end{aligned}
$$

It follows that

$$
M_{\delta}(x)=M_{x, a}+M_{x, 0} \frac{p M_{\delta, a}+q N_{-\delta,-b}}{1-p M_{\delta, 0}-q N_{-\delta, 0}} .
$$

Finally,

$$
M(x)=\lim _{\delta \downarrow 0} M_{\delta}(x)=M_{x, a}+M_{x, 0} \lim _{\delta \downarrow 0} \frac{p M_{\delta, a}+q N_{-\delta,-b}}{1-p M_{\delta, 0}-q N_{-\delta, 0}}, \quad \text { for } 0<x<a .
$$

The limit is obtained by using l'Hôpital's rule.

Now, as a function of $x$, the function $M_{x, a}$ satisfies the Kolmogorov backward equation

$$
\frac{1}{2} \sigma_{+}^{2} M_{x, a}^{\prime \prime}+\mu_{+} M_{x, a}^{\prime}=\alpha M_{x, a},
$$

subject to the boundary conditions

$$
\left.M_{x, a}\right|_{x=a}=1 \quad \text { and }\left.\quad M_{x, a}\right|_{x=0}=0 .
$$


Similarly,

$$
\frac{1}{2} \sigma_{+}^{2} M_{x, 0}^{\prime \prime}+\mu_{+} M_{x, 0}^{\prime}=\alpha M_{x, 0},
$$

subject to

$$
\left.M_{x, 0}\right|_{x=a}=0 \quad \text { and }\left.\quad M_{x, 0}\right|_{x=0}=1 .
$$

When $x$ is negative, we have

$$
\frac{1}{2} \sigma_{-}^{2} N_{x,-b}^{\prime \prime}+\mu_{-} N_{x,-b}^{\prime}=\alpha N_{x,-b},
$$

subject to

$$
\left.N_{x,-b}\right|_{x=-b}=1 \quad \text { and }\left.\quad N_{x,-b}\right|_{x=0}=0,
$$

and

$$
\frac{1}{2} \sigma_{-}^{2} N_{x, 0}^{\prime \prime}+\mu_{-} N_{x, 0}^{\prime}=\alpha N_{x, 0},
$$

subject to

$$
\left.N_{x, 0}\right|_{x=-b}=0 \quad \text { and }\left.\quad N_{x, 0}\right|_{x=0}=1 .
$$

We can easily solve the ordinary differential equations satisfied by the functions $M_{x, a}, M_{x, 0}$, etc. Let

$$
\begin{array}{ll}
\beta_{ \pm}=\frac{2 \mu_{ \pm}}{\sigma_{ \pm}^{2}}, & \lambda_{ \pm}=\sqrt{\beta_{ \pm}^{2}+\frac{8 \alpha}{\sigma_{ \pm}^{2}}} \\
\gamma_{1}^{ \pm}=\frac{-\beta_{ \pm}-\lambda_{ \pm}}{2}, & \gamma_{2}^{ \pm}=\frac{-\beta_{ \pm}+\lambda_{ \pm}}{2} .
\end{array}
$$

We find that

$$
\begin{aligned}
M_{x, a} & =\frac{\mathrm{e}^{\gamma_{1}^{+} x}-\mathrm{e}^{\gamma_{2}^{+} x}}{\mathrm{e}^{\gamma_{1}^{+} a}-\mathrm{e}^{\gamma_{2}^{+} a}}, & M_{\delta, a} & =\left.M_{x, a}\right|_{x=\delta}, \\
M_{x, 0} & =\frac{\mathrm{e}^{\gamma_{1}^{+}(x-a)}-\mathrm{e}^{\gamma_{2}^{+}(x-a)}}{\mathrm{e}^{-\gamma_{1}^{+} a}-\mathrm{e}^{-\gamma_{2}^{+} a}}, & M_{\delta, 0} & =\left.M_{x, 0}\right|_{x=\delta}, \\
N_{x,-b} & =\frac{\mathrm{e}^{\gamma_{1}^{-} x}-\mathrm{e}^{\gamma_{2}^{-} x}}{\mathrm{e}^{-\gamma_{1}^{-} b}-\mathrm{e}^{-\gamma_{2}^{-} b}}, & N_{-\delta,-b} & =\left.N_{x,-b}\right|_{x=-\delta}, \\
N_{x, 0} & =\frac{\mathrm{e}^{\gamma_{1}^{-}(x+b)}-\mathrm{e}^{\gamma_{2}^{-}(x+b)}}{\mathrm{e}^{\gamma_{1}^{-} b}-\mathrm{e}^{\gamma_{2}^{-} b}}, & N_{-\delta, 0} & =\left.N_{x, 0}\right|_{x=-\delta} .
\end{aligned}
$$

Then, using (6) and l'Hôpital's rule, we obtain the following proposition.

Proposition 1. The moment generating function of the random variable $T(x)$ defined in $(1)$ is

$$
\begin{aligned}
M(x)=M_{x, a}+M_{x, 0}[ & \left(p \frac{\gamma_{2}^{+}-\gamma_{1}^{+}}{\mathrm{e}^{\gamma_{1}^{+} a}-\mathrm{e}^{\gamma_{2}^{+} a}}+q \frac{\gamma_{1}^{-}-\gamma_{2}^{-}}{\mathrm{e}^{-\gamma_{1}^{-} b}-\mathrm{e}^{-\gamma_{2}^{-} b}}\right) \\
& \left.\times\left(p \frac{\gamma_{1}^{+} \mathrm{e}^{-\gamma_{1}^{+} a}-\gamma_{2}^{+} \mathrm{e}^{-\gamma_{2}^{+} a}}{\mathrm{e}^{-\gamma_{1}^{+} a}-\mathrm{e}^{-\gamma_{2}^{+} a}}-q \frac{\gamma_{1}^{-} \mathrm{e}^{\gamma_{1}^{-} b}-\gamma_{2}^{-} \mathrm{e}_{2}^{-} b}{\mathrm{e}^{\gamma_{1}^{-} b}-\mathrm{e}^{\gamma_{2}^{-} b}}\right)^{-1}\right],
\end{aligned}
$$

for $0<x<a$, where $M_{x, a}$ and $M_{x, 0}$ are given by (11) and (12), respectively. 
Remark 1. The value of $M(0)$ is deduced from (8) and (9). That is, $M(0)$ is the expression between the square brackets in (13). Furthermore, the formula for the case when $-b<x<0$ is obtained by replacing $M_{x, a}$ by $N_{x,-b}$, and $M_{x, 0}$ by $N_{x, 0}$ in (13).

Remark 2. If we take $a=b, p=q=\frac{1}{2}, \mu_{ \pm}=0$, and $\sigma_{ \pm}=\sigma$, we have $\gamma_{1}^{ \pm}=-\gamma_{2}^{ \pm}=\gamma:=$ $-\sqrt{2 \alpha} / \sigma$, and we retrieve the well-known formula

$$
\mathrm{E}\left[\mathrm{e}^{-\alpha T(x)}\right]=\frac{\mathrm{e}^{\gamma x}+\mathrm{e}^{-\gamma x}}{\mathrm{e}^{\gamma a}+\mathrm{e}^{-\gamma a}}=\frac{\cosh \gamma x}{\cosh \gamma a},
$$

which is valid for $-a \leq x \leq a$.

Remark 3. The function $M(x)$ in the case when there is a single barrier can be deduced from (13) by taking the limit as $b$ or $a$ tends to infinity.

\subsection{The probability of hitting $a$ before $-b$}

Let

$$
\pi_{a}(x)=\mathrm{P}[X(T(x))=a]
$$

and

$$
\pi_{a}^{\delta}(x)=\mathrm{P}\left[X\left(T_{\delta}(x)\right)=a\right] .
$$

Furthermore, let $\pi_{x, a}=\mathrm{P}[F]$ and $v_{x, 0}=\mathrm{P}\left[G^{\mathrm{c}}\right]$, where $F$ and $G$ are defined in (3). For $0<x<a$, we may write

$$
\pi_{a}^{\delta}(x)=\pi_{x, a}+\left(1-\pi_{x, a}\right) \pi_{a}^{\delta}(0),
$$

where

$$
\pi_{a}^{\delta}(0)=p \pi_{a}^{\delta}(\delta)+q \pi_{a}^{\delta}(-\delta)
$$

We have

$$
\pi_{a}^{\delta}(\delta)=\pi_{\delta, a}+\left(1-\pi_{\delta, a}\right) \pi_{a}^{\delta}(0)
$$

and

$$
\pi_{a}^{\delta}(-\delta)=v_{-\delta, 0} \pi_{a}^{\delta}(0)
$$

implying that

$$
\pi_{a}^{\delta}(x)=\pi_{x, a}+\left(1-\pi_{x, a}\right) \frac{p \pi_{\delta, a}}{1-p\left(1-\pi_{\delta, a}\right)-q \nu_{-\delta, 0}} .
$$

As a function of $x$, the function $\pi_{x, a}$ satisfies the following Kolmogorov backward equation:

$$
\frac{1}{2} \sigma_{+}^{2} \pi_{x, a}^{\prime \prime}+\mu_{+} \pi_{x, a}^{\prime}=0
$$

subject to

$$
\left.\pi_{x, a}\right|_{x=a}=1 \quad \text { and }\left.\quad \pi_{x, a}\right|_{x=0}=0 .
$$

We find that

$$
\pi_{x, a}=\frac{1-\mathrm{e}^{-\beta_{+} x}}{1-\mathrm{e}^{-\beta_{+} a}}, \quad \text { if } \mu_{+} \neq 0,
$$

and

$$
\pi_{x, a}=\frac{x}{a}, \quad \text { if } \mu_{+}=0 .
$$


Similarly,

$$
\frac{1}{2} \sigma_{-}^{2} v_{x, 0}^{\prime \prime}+\mu_{-} v_{x, 0}^{\prime}=0
$$

subject to

$$
\left.v_{x, 0}\right|_{x=0}=1 \quad \text { and }\left.\quad v_{x, 0}\right|_{x=-b}=0,
$$

which implies that

$$
\nu_{-\delta, 0}=\frac{\mathrm{e}^{\beta_{-} \delta}-\mathrm{e}^{\beta_{-} b}}{1-\mathrm{e}^{\beta_{-} b}}, \quad \text { if } \mu_{-} \neq 0,
$$

and

$$
\nu_{-\delta, 0}=\frac{b-\delta}{b}, \quad \text { if } \mu_{-}=0 .
$$

Hence (after a little bit of work), we obtain the following result.

Proposition 2. If $\mu_{ \pm} \neq 0$, then the probability $\pi_{a}(x)$ that the stochastic process $\{X(t), t \geq 0\}$, starting from $X(0)=x$, is absorbed at a is

$$
\pi_{a}(x)=\lim _{\delta \downarrow 0} \pi_{a}^{\delta}(x)=\pi_{x, a}+\left(1-\pi_{x, a}\right) \frac{p \beta_{+}\left(1-\mathrm{e}^{\beta_{-} b}\right)}{p \beta_{+}\left(1-\mathrm{e}^{\beta_{-} b}\right)+q \beta_{-}\left(\mathrm{e}^{-\beta_{+} a}-1\right)},
$$

for $0 \leq x<a$, where $\pi_{x, a}$ is given in (14).

In the case when $\mu_{+}=\mu_{-}=0$, we have

$$
\pi_{a}(x)=\frac{x}{a}+\left(1-\frac{x}{a}\right) \frac{p b}{p b+q a} .
$$

Remark 4. If $a=b, p=q=\frac{1}{2}, \mu_{ \pm}=\mu(\neq 0), \sigma_{ \pm}=\sigma$, and $\beta:=2 \mu / \sigma^{2}$, then

$$
\pi_{a}(x)=\frac{\mathrm{e}^{\beta a}-\mathrm{e}^{-\beta x}}{\mathrm{e}^{\beta a}-\mathrm{e}^{-\beta a}}, \quad \text { for }-a \leq x \leq a,
$$

which is correct. With $\mu=0$ and $\sigma=1$, we obtain

$$
\pi_{a}(x)=\frac{x+a}{2 a},
$$

which can also be deduced from

$$
\frac{1}{2} \pi_{a}^{\prime \prime}(x)=0, \quad \text { with } \pi_{a}(a)=1 \text { and } \pi_{a}(-a)=0 .
$$

Remark 5. If we set $x=0$ in (16), the general formula for $\pi_{a}(x)$, we retrieve the corresponding formula of Carlsund (2003).

Remark 6. The function $\pi_{a}(x)$ for $-b<x<0$ is given by

$$
\pi_{a}(x)=v_{x, 0} \pi_{a}(0) .
$$

Remark 7. If $\mu_{-}$is positive, then so is $\beta_{-}:=2 \mu_{-} / \sigma_{-}^{2}$. Taking the limit of $\pi_{a}(x)$ as $b$ tends to infinity, we find that $\pi_{a}(x)=1$, as expected. However, if $\mu_{-}$is negative, we find that

$$
\lim _{b \rightarrow \infty} \pi_{a}(x)=\pi_{x, a}+\left(1-\pi_{x, a}\right) \frac{p \beta_{+}}{p \beta_{+}+q \beta_{-}\left(\mathrm{e}^{-\beta_{+} a}-1\right)} .
$$

Therefore, it is not certain that the process will be absorbed (at $a$ ) in this case. 
Remark 8. To prove that $\pi_{a}(x)=\lim _{\delta \downarrow 0} \pi_{a}^{\delta}(x)$, as claimed in Proposition 2, we can compute first the probability of hitting $a / \delta_{+} x$ before $-b / \delta_{-} x$, starting from $x / \delta_{+} x$, for the signdependent Markov chain defined at the beginning of this section, and then find the limiting probability. In the case when $p_{ \pm}=\frac{1}{2}$, using the well-known results on the gambler's ruin problem, we may write (for $0 \leq x \leq a$ )

$$
\pi_{x / \delta_{+} x, a / \delta_{+} x}=\frac{x / \delta_{+} x}{a / \delta_{+} x}=\frac{x}{a} .
$$

It follows that

$$
\pi_{a / \delta_{+} x}\left(\frac{x}{\delta_{+} x}\right)=\frac{x}{a}+\left(1-\frac{x}{a}\right) \pi_{a \delta_{+} x}(0),
$$

where

$$
\pi_{a / \delta_{+} x}(0)=\pi_{a / \delta_{+} x}\left(\delta_{+} x\right) p+\pi_{a / \delta_{+} x}\left(-\delta_{-} x\right) q .
$$

Since

$$
\pi_{a / \delta_{+} x}\left(\frac{x}{\delta_{-} x}\right)=\left(1+\frac{x}{b}\right) \pi_{a / \delta_{+} x}(0),
$$

for $-b \leq x<0$, we find, taking the limit as $\delta_{+} x$ and $\delta_{-} x$ decrease to 0 simultaneously, that

$$
\pi_{a / \delta_{+} x}\left(\frac{x}{\delta_{+} x}\right) \rightarrow \frac{x}{a}+\left(1-\frac{x}{a}\right) \frac{p b}{p b+q a} .
$$

Hence, we can assert that (17) is valid.

Next, in the asymmetric case, we have

$$
\pi_{x / \delta_{+} x, a / \delta_{+} x}=\frac{1-\left(q_{+} / p_{+}\right)^{x / \delta_{+} x}}{1-\left(q_{+} / p_{+}\right)^{a / \delta_{+} x}},
$$

for $0 \leq x \leq a$. Furthermore, as mentioned previously, $p_{ \pm}-q_{ \pm}$must be equal to $\left(\mu_{ \pm} / \sigma_{ \pm}^{2}\right) \delta_{ \pm} x$, implying that

$$
\begin{aligned}
& \pi_{x / \delta_{+} x, a / \delta_{+} x} \\
& =\left(1-\left(\frac{2}{\left(\mu_{+} / \sigma_{+}^{2}\right) \delta_{+} x+1}-1\right)^{x / \delta_{+} x}\right)\left(1-\left(\frac{2}{\left(\mu_{+} / \sigma_{+}^{2}\right) \delta_{+} x+1}-1\right)^{a / \delta_{+} x}\right)^{-1} .
\end{aligned}
$$

Taking the limit as $\delta_{+} x$ decreases to 0 , we find that (see (10) and (14))

$$
\pi_{x / \delta_{+} x, a / \delta_{+} x} \rightarrow \frac{1-\mathrm{e}^{-\beta_{+} x}}{1-\mathrm{e}^{-\beta_{+} a}} .
$$

It is then rather straightforward to retrieve (16).

\subsection{The expected value of $T(x)$}

We will compute the expected value of $T(x)$ directly. We let $m(x)=\mathrm{E}[T(x)]$ and $m_{\delta}(x)=$ $\mathrm{E}\left[T_{\delta}(x)\right]$, and we define (see (3) and (4))

$$
\begin{aligned}
m_{x, a} & =\mathrm{E}\left[T_{x, a}^{+}\right] \mathrm{P}[F], \\
m_{x, 0} & =\mathrm{E}\left[T_{x, 0}^{+}\right] \mathrm{P}\left[F^{\mathrm{c}}\right], \\
n_{x,-b} & =\mathrm{E}\left[T_{x,-b}^{-}\right] \mathrm{P}[G], \\
n_{x, 0} & =\mathrm{E}\left[T_{x, 0}^{-}\right] \mathrm{P}\left[G^{\mathrm{c}}\right] .
\end{aligned}
$$


Then we may write (for $0<x<a$ )

$$
\begin{aligned}
m(x) & =\lim _{\delta \downarrow 0} m_{\delta}(x) \\
& =m_{x, a}+m_{x, 0}+\pi_{x, 0} \lim _{\delta \downarrow 0} \frac{p\left(m_{\delta, a}+m_{\delta, 0}\right)+q\left(n_{-\delta,-b}+n_{-\delta, 0}\right)}{1-p \pi_{\delta, 0}-q \nu_{-\delta, 0}} .
\end{aligned}
$$

Next, the function $M_{x, a}$ defined in (5) is given by

$$
\begin{aligned}
M_{x, a} & :=\mathrm{E}\left[\mathrm{e}^{-\alpha T_{x, a}^{+}}\right] \mathrm{P}[F] \\
& =: \mathrm{E}\left[\mathrm{e}^{-\alpha T_{x, a}^{+}}\right] \pi_{x, a} \\
& =\left\{1-\alpha \mathrm{E}\left[T_{x, a}^{+}\right]+\cdots\right\} \pi_{x, a} \\
& =\pi_{x, a}-\alpha m_{x, a}+\cdots
\end{aligned}
$$

Substituting this into the ordinary differential equation (7), we deduce that

$$
\frac{1}{2} \sigma_{+}^{2} m_{x, a}^{\prime \prime}+\mu_{+} m_{x, a}^{\prime}=-\pi_{x, a} .
$$

The function $\pi_{x, a}$ is given in (14) (for $\mu_{+} \neq 0$ ) and the boundary conditions are

$$
\left.m_{x, a}\right|_{x=a}=\left.m_{x, a}\right|_{x=0}=0 .
$$

Similarly, we may write (with $v_{x, 0}=\mathrm{P}\left[G^{\mathrm{c}}\right]$; see Section 2.2)

$$
\begin{array}{cc}
\frac{1}{2} \sigma_{+}^{2} m_{x, 0}^{\prime \prime}+\mu_{+} m_{x, 0}^{\prime}=-\pi_{x, 0}=-1+\pi_{x, a}, & \text { with }\left.m_{x, 0}\right|_{x=0, a}=0, \\
\frac{1}{2} \sigma_{-}^{2} n_{x, 0}^{\prime \prime}+\mu_{-} n_{x, 0}^{\prime}=-v_{x, 0}, & \text { with }\left.n_{x, 0}\right|_{x=0,-b}=0, \\
\frac{1}{2} \sigma_{-}^{2} n_{x,-b}^{\prime \prime}+\mu_{-} n_{x,-b}^{\prime}=-v_{x,-b}=-1+v_{x, 0}, & \text { with }\left.n_{x,-b}\right|_{x=0,-b}=0 .
\end{array}
$$

When $\mu_{ \pm} \neq 0$, we must solve an ordinary differential equation of the form

$$
h^{\prime \prime}(x)+\operatorname{ch}^{\prime}(x)=d+k \mathrm{e}^{-c x},
$$

where $c \neq 0, d$, and $k$ are constants, subject to $h(0)=0$ and $h(a)=0$ (or $h(-b)=0$ ). The functions $m_{x, a}, m_{x, 0}, n_{x, 0}$, and $n_{x,-b}$ can now be obtained by making use of the following proposition.

Proposition 3. The solution of (20) that satisfies the boundary conditions $h(0)=0$ and $h(a)=0$ is

$$
h(x)=\frac{d x}{c}-\frac{k x}{c} \mathrm{e}^{-c x}+\frac{1-\mathrm{e}^{-c x}}{1-\mathrm{e}^{-c a}}\left\{-\frac{d a}{c}+\frac{k a}{c} \mathrm{e}^{-c a}\right\} .
$$

Moreover, we replace $a$ by $-b$ if the boundary condition is $h(-b)=0$ rather than $h(a)=0$.

When $\mu_{+}=0$, we have (see (15))

$$
\frac{1}{2} \sigma_{+}^{2} m_{x, a}^{\prime \prime}=-\pi_{x, a}=-\frac{x}{a} .
$$

We find that

$$
m_{x, a}=\frac{\left(a^{2}-x^{2}\right) x}{3 a \sigma_{+}^{2}}, \quad \text { for } 0<x<a .
$$


In a similar way, we can compute $m_{x, 0}$ when $\mu_{+}=0$, and $n_{x, 0}$ and $n_{x,-b}$ when $\mu_{-}=0$. We find that

$$
\begin{aligned}
m_{x, 0}=\frac{\left(2 a^{2}+x^{2}\right) x}{3 a \sigma_{+}^{2}}-\frac{x^{2}}{\sigma_{+}^{2}}, & \text { for } 0<x<a, \\
n_{x, 0}=\frac{-\left(2 b^{2}+x^{2}\right) x}{3 b \sigma_{-}^{2}}-\frac{x^{2}}{\sigma_{-}^{2}}, & \text { for }-b<x<0, \\
n_{x,-b}=\frac{-\left(b^{2}-x^{2}\right) x}{3 b \sigma_{-}^{2}}, & \text { for }-b<x<0 .
\end{aligned}
$$

Making use of these formulae, we find that, when $\sigma_{ \pm}=1$ and $a=b$, the function $m(x)$ is given by (see (18))

$$
m(x)=a^{2}-x^{2}, \quad \text { for } 0<x<a .
$$

Actually, this solution is valid for $x \in[-a, a]$.

Now, in the case of the standard Brownian motion, $m(x)$ satisfies the ordinary differential equation

$$
\frac{1}{2} m^{\prime \prime}(x)=-1, \quad \text { for }-a<x<a,
$$

subject to the boundary conditions $m( \pm a)=0$. We find at once that the function obtained above is indeed the solution to this ordinary differential equation that satisfies the appropriate boundary conditions.

Remark 9. Instead of proceeding as above, we can use

$$
m_{x, a}=-\lim _{\alpha \downarrow 0} \frac{\partial}{\partial \alpha} M_{x, a}
$$

and the corresponding formulae for $m_{x, 0}, n_{x, 0}$, and $n_{x,-b}$. This will work but is quite tedious. For instance, if we take $\mu_{+}=0$ and $\sigma_{+}=1$, then the function $M_{x, a}$ becomes

$$
M_{x, a}=\frac{\sinh \sqrt{2 \alpha} x}{\sinh \sqrt{2 \alpha} a}
$$

and we find (after some work) that

$$
-\lim _{\alpha \downarrow 0} \frac{\partial}{\partial \alpha} M_{x, a}=\frac{\left(a^{2}-x^{2}\right) x}{3 a},
$$

which agrees with the result (21), with $\sigma_{+}=1$. Note that this justifies the boundary condition (see (19)) $\left.m_{x, a}\right|_{x=0}=0$.

Remark 10. To prove that $m(x)=\lim _{\delta \downarrow 0} m_{\delta}(x)$, we can use the formulae in Feller (1968, p. 348) for the expected duration of the game in the gambler's ruin problem. In the symmetric case, this expected duration, denoted by $D$, is

$$
D(x)=x(a-x), \quad \text { for } 0<x<a .
$$

The corresponding formula for the sign-dependent random walk (with $\delta_{ \pm} x=\delta x$ ) between $-a / \delta x$ and $a / \delta x$ is

$$
D\left(\frac{x}{\delta x}\right)=\left[-\left(\frac{x}{\delta x}\right)^{2}+A+B\left(\frac{x}{\delta x}\right)\right] \delta t, \quad \text { for }-\frac{a}{\delta x}<x<\frac{a}{\delta x},
$$


where $A$ and $B$ are chosen to satisfy the boundary conditions

$$
D\left( \pm \frac{a}{\delta x}\right)=0 .
$$

We find that

$$
B=0 \quad \text { and } \quad A=\frac{a^{2}}{(\delta x)^{2}} ;
$$

thus, when $(\delta x)^{2}=\delta t$, taking the limit as $\delta x$ decreases to 0 yields

$$
D\left(\frac{x}{\delta x}\right) \rightarrow a^{2}-x^{2}
$$

and we retrieve (22) for $m(x)$.

In the asymmetric case, we could also use the appropriate expressions found in Feller (1968) to compute the value of $m(x)$, in the same way as we did for the probability $\pi_{a}(x)$.

\section{Conclusion}

We have generalized the classical formula giving the moment generating function of the first passage time $T(x)$ out of the interval $(-b, a)$ for a one-dimensional Wiener process. In our case, the infinitesimal parameters of the process $\{X(t), t \geq 0\}$ are constants that may depend on the sign of $X(t)$.

To further generalize the results obtained in this note, we could assume that the infinitesimal parameters $\mu$ and $\sigma^{2}$ can take more than two different values each. We could also consider the case when $\mu$ and $\sigma^{2}$ are both random variables in distinct parts of the real line. If $\mu$ and $\sigma^{2}$ are random variables such that each takes a single value over the whole real line, then we simply have to use conditional expectation to obtain the moment generating function of $T(x)$.

We can also, of course, try to derive the formulae that correspond to the ones presented here for other diffusion processes, such as the Ornstein-Uhlenbeck process. Although these problems are not very complicated mathematically, the explicit solutions, especially in the case of the Ornstein-Uhlenbeck process, will probably be quite involved.

\section{Acknowledgements}

This research was supported by the Natural Sciences and Engineering Research Council of Canada. The author also wishes to thank the referee and the Editor who handled this paper for constructive comments.

\section{References}

CARlsund, A. (2003). Cover times, sign-dependent random walks, and maxima. Doctoral Thesis, Royal Institute of Technology, Stockholm. Summary available at http://www.math.kth.se/matstat/fofu/reports/carlsundspikblad.pdf.

Chong, K. S., Cowan, R. And Holst, L. (2000). The ruin problem and cover times of asymmetric random walks and Brownian motions. Adv. Appl. Prob. 32, 177-192.

Cox, D. R. AND Miller, H. D. (1965). The Theory of Stochastic Processes. Methuen, London.

Feller, W. (1968). An Introduction to Probability Theory and its Applications, Vol. I, 3rd edn. John Wiley, New York. Harrison, J. M. And Shepp, L. A. (1981). On skew Brownian motion. Ann. Prob. 9, 309-313.

Itô, K. And McKean, H. P., JR. (1974). Diffusion Processes and their Sample Paths. Springer, Berlin.

Ovaskainen, O. ANd CoRnell, S. J. (2003). Biased movement at a boundary and conditional occupancy times for diffusion processes. J. Appl. Prob. 40, 557-580. 